Review Article

\title{
An Improved Whale Algorithm for Setting Standard Scheduled Block Time Based on the Airline Fairness
}

\author{
Qian Wang, ${ }^{1}$ Yong Tian $\mathbb{D}^{1},{ }^{1}$ Lili Lin, ${ }^{2}$ Ratnaji Vanga, ${ }^{3}$ and Lina $\mathrm{Ma}^{1}$ \\ ${ }^{1}$ College of Civil Aviation, Nanjing University of Aeronautics and Astronautics, Nanjing 211100, China \\ ${ }^{2}$ Yangzhou Collaborative Innovation Research Academy of Shenyang Aircraft Design \& Research Institute, \\ Shenyang 225002, China \\ ${ }^{3}$ Department of Industrial Systems Engineering \& Management, National University of Singapore, Singapore \\ Correspondence should be addressed to Yong Tian; tianyong@nuaa.edu.cn
}

Received 25 May 2020; Revised 9 August 2020; Accepted 21 September 2020; Published 9 October 2020

Academic Editor: Fabio Tramontana

Copyright (C) 2020 Qian Wang et al. This is an open access article distributed under the Creative Commons Attribution License, which permits unrestricted use, distribution, and reproduction in any medium, provided the original work is properly cited.

\begin{abstract}
Standard scheduled flight block time (SBT) setting is of great concern for Civil Aviation Administration of China (CAAC) and airlines in China. However, the standard scheduled flight block times are set in the form of on-site meetings in practice and current literature has not provided any efficient mathematical models to calculate the flight block times fairly among the airlines. The objective of this paper is to develop and solve a mathematical model for standard SBT setting with consideration of both fairness and reliability. We use whale optimization algorithm (WOA) and an improved version of the whale optimization algorithm (IWOA) to solve the SBT setting problem. A novel nonlinear update equation of convergence factor for random iterations is used in place of the original linear one in the proposed IWOA algorithm. Experimental results show that the suggested approach is effective, and IWOA performs better than WOA in the concerned problem, whose solutions are better compared to the flight block times released by CAAC. In particular, it is interesting to find that MSE, RMSE, MAE, MAPE and Theil of the reliability in $60 \%-70 \%$ range are always the smallest and the average fairness of airlines is better than that of $60 \%-75 \%$ range. The model and solving approach presented in this article have great potential to be applied by CAAC to determine the standard SBTs strategically.
\end{abstract}

\section{Introduction}

According to the statistical report published by CAAC, the number of flights in China increased by 3.093 million (36.1\%) from 2015 to 2019. The rapid growth of air transportation demand has led to a serious issue of flight delays. The statistics show that there were 2.358 million departure flights in China out of 18.8 million all over the world and China ranked second in terms of the number of flights in the first half of 2019. However, China ranked eighth in the punctuality rate rankings.

It is recognized that the factors affecting on-time performance could be mitigated by adjustments in standard scheduled block time (SBT), which is defined as the time interval between scheduled gate departure at the departure airport and scheduled gate arrival at the arrival airport for a given flight [1]. In principle, a higher on-time performance could be achieved by increasing the standard SBTs for these flights for airlines operating flights in highly congested or delay-prone conditions $[2,3]$.

Figure 1 illustrates SBT and flight block time decomposition, including flight taxi-out time, flight air time, and flight taxi-in time $[3,4]$. The time between scheduled departure time and actual departure time is defined as flight departure delay time. Similarly, the time between scheduled arrival time and actual arrival time is defined as flight arrival delay time.

Standard SBT is an important reference for Operation Monitoring Center of CAAC to set strategic flight schedule twice per year for all flights (once for the winter and once for the summer scheduling season) $[1,4]$. Standard SBT needs to be adjusted yearly with the development of air traffic. Actual block time is uncertain because of various factors such as adverse weather condition and airport/airspace congestion 


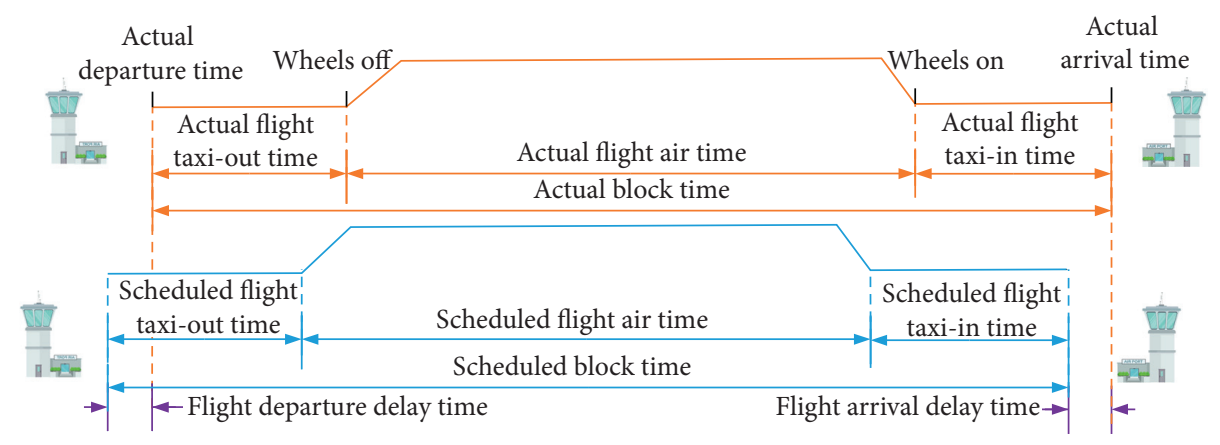

FIgURE 1: SBT and flight block time decomposition.

[5]. Accordingly, it creates a "too much" versus "too little" trade-off in the scheduling decision, similar to typical newsvendor model. If too much standard SBT is allocated to a flight, it creates leftover inventory (overage) costs for the airline such as lower utilization rate of aircraft and pilot compensation. On the contrary, it is likely to exceed the standard SBT resulting in delay and shortage (underage) costs for the airline such as spending for dealing with dissatisfied customers, overtime costs, and so forth. More importantly, a lower punctuality rate will cause huge damage to the image of civil aviation, thereby reducing the competitiveness with road traffic.

The Operation Monitoring Center of CAAC publishes the standard SBT table every year on preflight plan management system on http://www.pre-flight.cn/. The table contains standard SBTs between airport pairs that have regularly scheduled flights between them. The flights are categorized by air season, departure-arrival airports pair, and aircraft type. Air season 7 includes summer and autumn and air season 8 includes winter and spring. For the same departure and landing airports, different aircraft types and air seasons may have different standard SBTs.

Table 1 gives an example of standard SBTs from the table published by CAAC. M0.8-0.89 represents aircraft with Mach number from 0.8 to 0.89 , for example, A330, A380, and B787-8. M0.7-0.79 represents aircraft with Mach number from 0.7 to 0.79, for example, A300, A320, and B737. For example, the standard SBT of airport pair from Beijing Capital International Airport (ZBAA) to Guangzhou Baiyun International Airport (ZGGG) is 200 minutes from March 25, 2018, to October 27, 2018, using M0.8-0.89 aircraft type, while that of the opposite direction is 190 minutes.

At the strategic level, there is a proactive schedule coordination process (more than 6 months before operations) with bilateral communication between the Operation Monitoring Center of CAAC and airlines [1]. The Operation Monitoring Center of CAAC holds a civil aviation advance flight planning meeting that convenes the employees of the Marketing and Sales Committee (MSC) from all airlines in China to adjust the standard SBTs. The meeting usually takes 2 to 3 days. The Operation Monitoring Center compares the proposed standard SBTs mainly based on the analysis of the median, mean, and standard value of the historical flight data and opinions made by each airline. The standard SBTs must be accepted by all airlines. The method of adjusting standard SBTs by centralized on-site discussion and referring historical data is highly subjective and inefficient and can be written as "inefficient way of resource utilization." Due to this, we propose to overcome these disadvantages by using optimization methods. To the best of our knowledge, there is no such research that uses mathematical modelling methods to set SBTs.

This paper aims to propose an optimization method to set SBTs of all airport pairs using various aircraft types in different air seasons more efficiently and reliably. The method may be useful to CAAC, which is the chief of the civil aviation advance flight planning conference in providing optimal decisions. This method establishes the mathematical model of the standard SBTs setting. There is no prior research in addressing the way to optimize standard SBTs with consideration of the fairness of airlines, which is established by this article.

Thus, this article focuses on addressing the following questions:

(i) How is actual flight block time reliability performance affected by the standard SBTs? What is about flight taxi-in time and flight taxi-out time?

(ii) How to establish a mathematical model to adjust standard SBTs based on the historical operation statistics considering the fairness of airlines?

(iii) What is the effect of different reliability ranges on the optimization results of SBTs? What is the best range of reliability?

The remainder of this paper is organized as follows. Section 2 reviews the background information regarding standard SBT and block time reliability (BTR). In Section 3, we analyze how actual flight block time reliability performance is affected by the standard SBTs based on the analysis of historical flight data of all airlines. Section 4 presents our proposed standard SBT optimal model based on the BTR, which also considers the fairness of airlines in China. In addition, WOA and IWOA algorithms are chosen to solve the optimization problem of standard SBT for comparison. In Section 5, we illustrate its application to the case study followed by the discussion of main results of the standard SBTs of all flights. In addition, we analyze evaluation metrics of different reliability ranges to seek out the best range of reliability. Finally, conclusions and suggestions for further research are presented in Section 6. 
TABle 1: Example of standard block times.

\begin{tabular}{llcc}
\hline \multirow{2}{*}{ Departure-arrival airports pair } & \multirow{2}{*}{ Date } & \multicolumn{2}{c}{ SBT (min) } \\
& & Aircraft type 7 M0.8-0.89 & Aircraft type 8 M0.7-0.79 \\
\hline \multirow{2}{*}{ ZBAA <->ZGGG } & March 25, 2018-October 27, 2018 (air season 7) & $200 / 190$ & $205 / 195$ \\
& October 28, 2018-March 30, 2019 (air season 8) & $205 / 185$ & $220 / 190$ \\
\hline \multirow{2}{*}{ ZSAM<->ZSHC } & March 25, 2018-October 27, 2018 (air season 7) & $100 / 95$ & $95 / 100$ \\
& October 28, 2018-March 30, 2019 (air season 8) & $90 / 100$ & $95 / 105$ \\
\hline
\end{tabular}

\section{Literature Review}

In this section, we present some of the literature of standard SBT and flight block time reliability. Firstly, we discuss research papers related to the relationship of standard SBT and on-time performance. Then, we present the fairness metric of slot scheduling, which is similar to flight block time scheduling and reliability metric after the first part, and the research gaps are summarized in the end.

Recent studies have examined the contribution of standard SBT setting to airline on-time performance and have proposed several models for understanding standard SBT-setting behavior. The SBT is close to the mean travel time. Increasing standard deviation results in a decrease in the SBT, as coefficients on the standard deviation of block times are negative [6]. On-time arrival probability depends significantly on the duration of standard SBTs. The overall on-time arrival percentage for civil aviation increases by $25.5 \%$ when the scheduled block time is added 15 minutes [5]. After this research, Kang and Hansen [2] estimated a correlated mixed logit model in which the choice set consists of different possible standard SBTs adjustments and the attributes were the projected scheduled reliability metrics that would result from the adjustment. They found that many airlines were willing to increase standard SBTs from 0.38 to 0.54 minutes to gain an increase in on-time performance of $1 \%$ by estimating the model for five different airlines in the United States. It has been found that standard SBTs depend mostly on the inner right tail and less on the far-right tail by Hao and Hansen [4]. Then, Wang et al. [1] quantified the importance of factors that decision-makers in China and USA consider when setting SBTs in the form of a multiple linear regression model. They found that a unit increased in the lower taxi-out time increased SBT more than a unit increased in the higher taxi-out time did.

Standard SBTs are important references to the flight schedule and operation such as crew scheduling, flight slot scheduling, and aircraft rotations. The process of scheduled flight block time setting is similar to that of strategic slot scheduling [7]. On the one hand, both consider decisions made twice per year. On the other hand, all stakeholders must participate in the formulation process and accept the final schedule. Standard SBT-setting research field is less intensive and extensive compared to slot scheduling research field, especially the idea of considering fairness in the model. The fairness has been introduced as a criterion for slot allocation in the context of congestion pricing by Andreatta and Lulli [8]. In particular, the notion of fairness has been introduced by Pellegrini et al. [9] in slot scheduling decisions at the network level by considering the maximum cost of missed allocation and the maximum total displacement costs of all considered airlines across all airports. Further, the fairness objective is defined based on a proportionality metric, which postulates that the total schedule displacement of an airline should be proportional to the number of slots it has requested [10].

The reliability in transportation mainly refers to the unpredictable variations in travel time and is thus directly related to uncertainty of travel time. The increase in air traffic and technology development comes with a greater expectation of reliable travel from the passenger perspective [11]. The travel time reliability is the variations in journey time that travellers cannot predict in ground transportation [12]. In other words, it presents the temporal uncertainty experienced by travellers in their movements between the origin and destination of the trip.

The primary basis for choosing standard SBT for a flight is the flight block time reliability [1]. The idea of reliability was first introduced from ground transportation to air transportation by Hao and Hansen [4]. Similarly, they firstly proposed the concept of block time reliability. Another important contribution by them is the theoretical model with the percentile statistics of the actual flight time, which estimates impact of historical block time distribution on SBT, that is, the reflection of BTR. BTR is defined as the proportion of flights whose block times do not exceed the standard SBTs $[1,4]$. In other words, the BTR for a certain categorization is the percentage of realized flights whose block times are shorter than or equal to the SBT.

In the above studies, various empirical models of standard SBTs setting are proposed to analyze the SBTsetting behavior and examine the contribution of standard SBTs setting to airline on-time performance. Furthermore, Chinese airlines sometimes adjust their scheduled block times based on operational experience. As per our knowledge, none of the studies establish an optimal model to adjust standard SBTs objectively. Accordingly, although BTR has been considered as an important conference in the procedure of scheduled block time setting, how it effects the results has not been studied.

This paper contributes to the flight block time scheduling by the following: (i) exploring the influence of standard SBTs on reliability by analyzing the historical flight data, (ii) introducing a new single-objective optimization model that considers the fairness objective and the flight block time reliability constraints and can adjust standard SBTs for the two seasons, (iii) using improved WOA algorithm to optimize standard SBTs, and (iv) investigating the appropriate 
range of reliability by analyzing evaluation metrics of different reliability ranges.

\section{An Analysis of Flight Block Time Reliability}

In this section, we analyze how the flight block time reliability, flight taxi-out time reliability, and flight taxi-in time reliability change with their different standards, respectively.

In this paper, the potential for changes in SBTs with the change of BTR is one of the major points of focus. To understand this, we first study the impact of the change in standard SBTs, the scheduled flight taxi-out time and the scheduled flight taxi-in time on BTR, and the flight taxi-out time reliability and the flight taxi-in time reliability, respectively. Flight block time reliability, flight taxi-in time reliability, and flight taxi-out time reliability are defined as follows [3].

$$
\begin{aligned}
R_{\text {block }} & =P\left\{t_{\text {block }} \leq T_{\text {block }}\right\}, \\
R_{\text {taxi-out }} & =P\left\{t_{\text {taxi-out }} \leq T_{\text {taxi-out }}\right\}, \\
R_{\text {taxi-in }} & =P\left\{t_{\text {taxi-in }} \leq T_{\text {taxi-in }}\right\} .
\end{aligned}
$$

Equations (1) to (3) estimate the flight block time reliability $R_{\text {block }}$, flight taxi-in time reliability $R_{\text {taxi-in }}$, and flight taxi-out time reliability, respectively. Equation (1) is used to calculate $R_{\text {block }}$, where $t_{\text {block }}$ is actual flight block time and $T_{\text {block }}$ is scheduled block time. $R_{\text {taxi-out }}$ is calculated by equation (2), in which $t_{\text {taxi-out }}$ is actual flight taxi-out time and $T_{\text {taxi-out }}$ is scheduled flight taxi-out time. Equation (3) is used to calculate $R_{\text {taxi-in }}$, and $t_{\text {taxi-in }}$ is actual flight taxi-in time and $T_{\text {taxi-in }}$ is scheduled flight taxi-in time.

We obtained the historical flight data of the operating flights in China from March 2018 to March 2019 from the CAAC. We determined the changes in reliability of the flight block time, taxi-in time, and taxi-out time when the standard SBT, standard scheduled flight taxi-out time, and standard scheduled flight taxi-in time increase (or the actual flight block time, actual flight taxi-out time, and actual taxi-in time decrease), respectively. These results are shown in Figures 2 and 3 .

Figure 2(a) is a set of detailed 3D graphs showing different flight routes against different reliabilities. Similarly, Figures 2(b) and 2(c) show different airports against different flight taxi-out time reliabilities and flight taxi-in reliabilities in detail. As mentioned in Section 1, we can observe the fact that different aircraft types and seasonal factors affect the standard SBTs of an airport. The abscissa of Figure 3 is the minutes that SBTs, scheduled flight taxi-out time increase. For example, the first point of flight block time in Figure 3 indicated that the average BTR of all flight routes is $43.44 \%$ under the standard SBTs, while the second point in blue color indicates that the mean reliability is increased to $46.56 \%$ when the SBTs are reduced by 1 minute. Similarly, the points in orange and blue express similar observations that the reliability increases with the decrease in SBTs.

According to Figure 3, we can see that the flight block time reliabilities increase by $33.61 \%$ and $57.07 \%$ with the standard times increasing by 5 minutes and 10 minutes (or the actual times decreasing by 5 minutes and 10 minutes), respectively. By comparing the results of flight taxi-out time and taxi-in time, it is found that the proportion of flight taxiout reliability increases more significantly with the increase of scheduled time compared to flight taxi-in time. The flight taxi-out time reliability increases from $76.54 \%$ to $84.09 \%$, and the flight taxi-in time reliability increases from $89.05 \%$ to $89.31 \%$ when both scheduled times increase by 5 minutes. Obviously, the reliability of flight taxi-out time improves by $9.85 \%$, while the reliability of the flight taxi-in time only improves by $0.29 \%$. As seen in Figure 3, when the scheduled flight taxi-out time increases by 1 minute (or the actual flight taxi-out time decreases by 1 minute), the reliability increases by $3.40 \%$. The above analyses show that, on the one hand, the reliability of flight taxi-in time is higher than that of flight taxi-out time, which is in line with the results of Tian et al. [3]. On the other hand, there is a lot of space for flight taxiout reliability to improve based on reducing the actual flight taxi-out time at the airport.

\section{Flight Block Time Optimization}

In this section, we first propose a mathematical model to set standard SBTs of all airport pairs considering the fairness of history operating airlines. This mathematical model is developed for setting the standard SBT scheduling. Further, we present WOA and improved WOA (IWOA) to solve this problem.

4.1. Formulation. The following notations are presented for the mathematical formulation of the proposed standard SBT-setting model.

Sets

Q: Set of airlines

$G$ : Set of airport pairs

$J$ : Set of aircraft types

$R$ : Set of air seasons

$T$ : Set of standard SBTs

$M_{q}$ : Set of difference between the old standard SBTs and the new requested time requested by airline $q$

$M$ : Set of differences requested by all airlines

$H$ : Set of historical flight block times.

Parameters

$t_{q}^{g, j, r}$ : Requested flight block time by airline $q$ for airport pair $g$, air season $j$, and aircraft type $r$

$t_{h}^{g, j, r}$ : Historical flight block time for airport pair $g$, air season $j$, and aircraft type $r$

$\underline{\sigma}$ : Lower limit of flight block time reliability constraint $\bar{\sigma}$ : Upper limit of flight block time reliability constraint.

Decision variables

$x_{m}^{t^{g, j, r},} x_{m}^{t^{g, j, r}}=1$, if difference $m$ is allocated to the requested time $t$; otherwise, $x_{m}^{t^{g, j, j}}=0$.

Function 


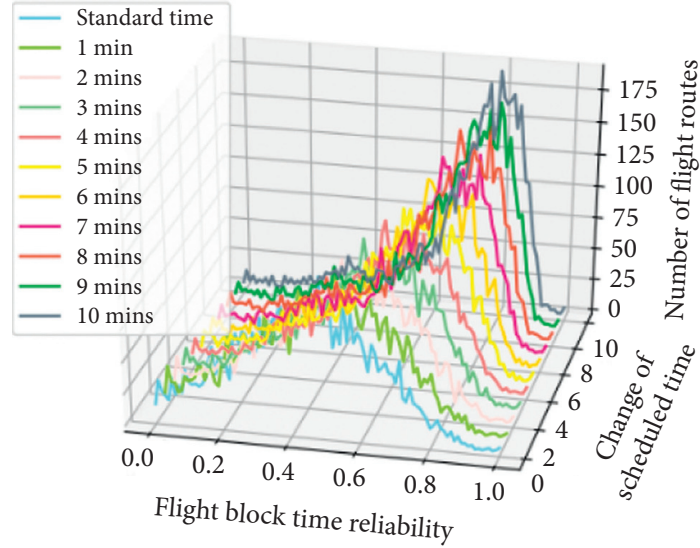

(a)

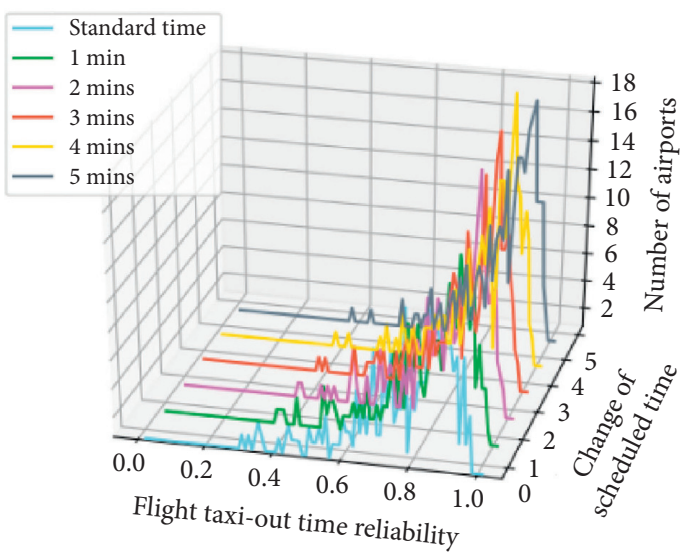

(b)

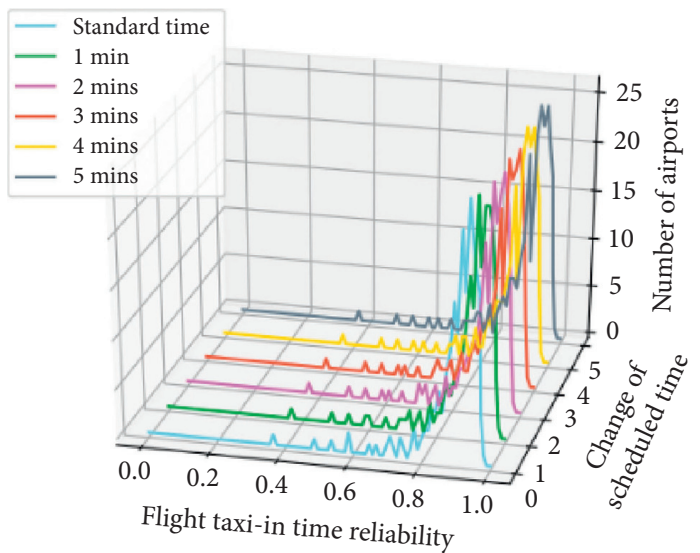

(c)

Figure 2: Reliabilities changes with different standard scheduled times. (a) Flight block time. (b) Flight taxi-out time. (c) Flight taxi in time.

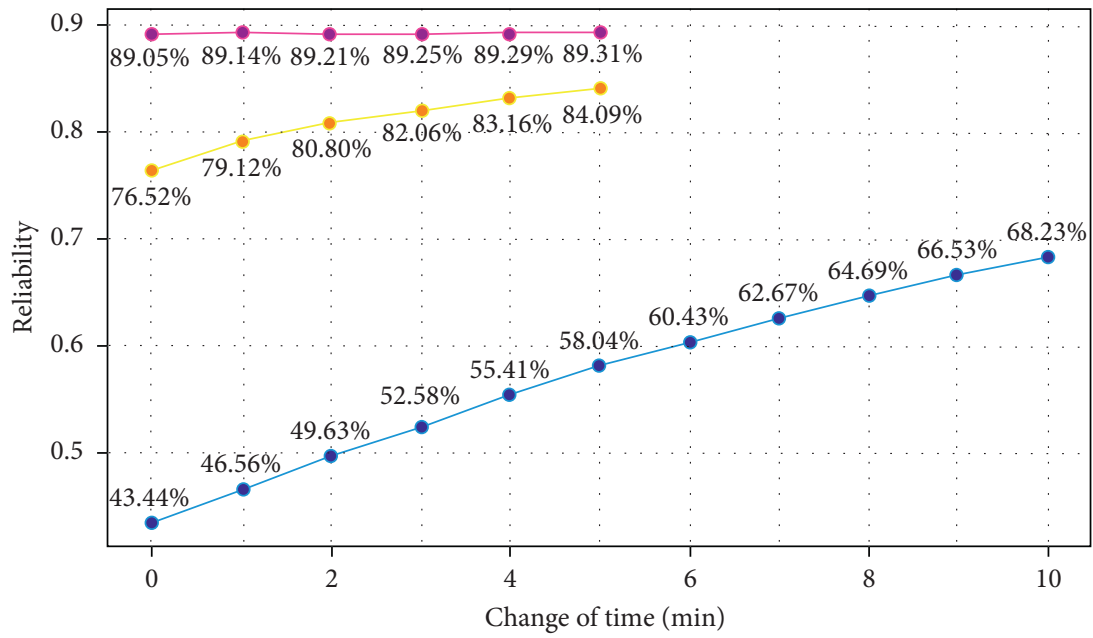

-- Flight block time

- Flight taxi-out reliability

$\longrightarrow$ Flight taxi-in reliability

FIGURE 3: Reliability of flight block time, flight taxi-in time, and flight taxi-out time with the change of standard times, respectively. 
$\rho_{q}:$ Fairness metric of airline $q$

$R^{g, j, r}$ : Flight block time reliability for airport pair $g$, air season $j$, and aircraft type $r$.

4.1.1. Allocated Displacement. Standard SBTs allocated displacement $f_{m}$ is defined as the difference between the allocated and historical flight block times for airport pair $g$, air season $j$, and aircraft type $r$. The allocated displacement of a difference $m$ is expressed by equation (4) mathematically.

$$
f_{m}=\sum_{h \in H} \sum_{t g, j, r \in T}\left|t^{g, j, r}-t_{h}^{g, j, r}\right| x_{m}^{t^{g, j, r}}, \quad \forall m \in M, g \in G, j \in J, r \in R .
$$

Based on the above equation, the total difference between the allocated and historical flight block times of an airline $(q)$ is expressed by equation (5), while the total allocated displacement of all flights for total airlines is computed using equation (6).

$$
\begin{aligned}
& d_{q}=\sum_{m \in M_{q}} f_{m}, \quad \forall q \in Q, \\
& D_{q}=\sum_{q \in Q} \sum_{m \in M_{q}} f_{m} .
\end{aligned}
$$

4.1.2. Requested Displacement. Standard SBTs requested displacement is defined as the difference between the requested and historical flight block times. Mathematically, it is expressed by the following equation:

$$
f_{m}^{\prime}=\sum_{h \in H} \sum_{t^{g, j, r} \in T}\left|t_{q}^{g, j, r}-t_{h}^{g, j, r}\right|, \quad \forall m \in M, \forall g \in G, j \in J, r \in R .
$$

Correspondingly, equation (8) expresses the total requested displacement, while equation (9) is used to calculate the requested displacement of all airlines.

$$
\begin{aligned}
d_{q}^{\prime} & =\sum_{m^{\prime} \in M_{q}} f_{m}^{\prime}, \quad \forall q \in Q, \\
D_{q}^{\prime} & =\sum_{q \in Q} \sum_{m^{\prime} \in M_{q}} f_{m}^{\prime}
\end{aligned}
$$

4.1.3. The Fairness Metric. The concept of scheduling displacement proportionally to requests has been proposed in the context of the Ground Delay Program [13]. Further, the concept was adopted to slots scheduling decision-making context [10]. In this paper, we adopt it to standard SBTsetting context for the first time. The total allocated displacements of an airline should be proportional to the requested displacement. The mathematical expression of the proposed fairness metric is as follows:

$$
\rho_{q}=\frac{\left(d_{q} / D\right)}{\left(d_{q}^{\prime} / D_{q}^{\prime}\right)}, \quad \forall q \in Q
$$

The denominator in the above formula is the proportion of standard SBTs requests by an airline $(q)$, while the numerator is the proportion of standard SBTs allocated to it $(q)$. Based on the above fairness indicator, the definition can be concluded as follows [10]: an airline is fairly treated if $\rho_{q}=1.0$; an airline is a favored airline if $\rho_{q}<1.0$; an airline is a disfavored airline if $\rho_{q}>1.0$.

Based on the proposed fairness metric, all airlines are fairly treated when $\rho_{q}=1, \forall q \in Q$. However, due to the difference in airline capability and the fact that a certain flight route is always operated by more than one airline, different airlines have different operating hours for the same flight route. Actual flight block time is uncertain because of various reasons such as weather conditions and airport/ airspace congestion. In this way, perfect flight block time scheduling for all airlines cannot be achieved. An alternative way of dealing with the fairness issue is to compare the displacement of a given airline with the displacement of other airlines (relative as opposed to absolute fairness). Accordingly, the fairness objective is proposed as minimizing the maximum distance between the fairness metric of an airline and the average fairness metric of all airlines. We propose the following mathematical model:

$$
\min z(x)=\left\{\max \left|\rho_{q}-\frac{\sum_{q^{\prime} \in Q} \rho_{q^{\prime}}}{|Q|}\right|, q \in Q\right\},
$$

subject to

$$
\begin{array}{r}
\underline{\sigma} \leq R^{g, j, r} \leq \bar{\sigma}, \quad \forall g \in G, j \in J, r \in R, \\
\sum_{t^{g, j, r} \in T} x_{m}^{t^{g, j, r}}=1, \quad \forall m \in M, g \in G, j \in J, r \in R, \\
x_{m}^{t^{g, j, r}} \in\{0,1\}, \quad \forall m \in M, g \in G, j \in J, r \in R .
\end{array}
$$

Here, equation (11) is the objective function which ensures that the worst case of unfairness differs as little as possible from the average fairness by minimizing the maximum distance between the fairness metric of an airline and the average fairness metric of all airlines. Equation (12) requests that the reliability of flight block time for airport pair $g$, air season $j$, and aircraft type $r$ is supposed to be in the range from $\underline{\sigma}$ to $\bar{\sigma}$. Its calculation formula refers to equation (1) in Section 3. Equation (13) stipulates that every flight block time of flight route must be allocated to one and only one time period. Equation (14) is a binary constraint on the decision variable. This mathematical model is similar to the allocation models that are NP-hard and thus it is difficult to solve it using solvers in a reasonable time. Generally, metaheuristics are used to get a good enough solution in a quick time [14]. In this paper, we propose a metaheuristic algorithm named improved whale optimization algorithm (IWOA), which optimizes the standard SBTs. 
4.2. Solving the Flight Block Time Optimization Model. This section aims to introduce the theory related to the standard whale optimization algorithm (WOA). The improved WOA (IWOA) is then proposed.

4.2.1. WOA. WOA is a new evolutionary computation algorithm proposed by Mirjalili and Lewis [15], which mimics the bubble-net hunting technique of humpback whales for global optimization problem [16]. Global optimization process is often divided into two subprocesses: exploration and exploitation. WOA is proved to be very competitive with metaheuristic optimizers and superior over conventional techniques. Mirjalili and Lewis [15] tested WOA with six constrained engineering design problems. The results show that the WOA algorithm outperforms particle swarm optimization (PSO) and gravitational search algorithm (GSA) in average and requires a smaller number of analyses (function evaluation). Moreover, it also has less error than GA, which is very important in fault detection and finding its location in the fault location estimation [17]. The humpback whales use the bubble-net hunting technique to encircle and catch their preys, which is also considered as the core intelligent mechanism used by the algorithm. WOA has been applied in many fields to solve practical problems. For instance, WOA is used to optimize the renewable resource configuration to reduce the wastage of the distribution system [18]. Olive et al. [19] applied an improved WOA to estimate parameter of photovoltaic cell.

(1) Searching and Encircling Prey (Exploration Phase). The humpback whales' attempts to update their positions are as follows:

$$
\begin{aligned}
D & =\left|C \cdot X_{\text {rand }}-X(t)\right|, \\
X(t+1) & =X_{\text {rand }}-\text { A.D }, \\
\mathrm{A} & =2 a \cdot r-a, \\
C & =2 . r,
\end{aligned}
$$

where $X_{\text {rand }}$ is randomly selected from the current population. $a$ is distance control parameter to balance the ability of exploration and exploitation, and it is linearly decreased from 2 to 0 over the course of iterations (in both exploration and exploitation phases) in WOA [15]. The distance of $r$ is a random vector in $[1,0]$ and $t$ is the current iteration.

$$
\begin{aligned}
D & =\left|C \cdot X^{*}(t)-X(t)\right|, \\
X(t+1) & =X^{*}(t)-\text { A.D, }
\end{aligned}
$$

where $X^{*}$ is the best solution obtained so far. If $|A| \geq 1$ the humpback whales balance between searching prey using equations (15) and (16); otherwise, the encircling prey using equations (19) and (20). WOA has different parameters to control the behavior of the population, and the main advantage of it is its capability in reproducing the mechanism lying behind the chase for prey.
(2) Bubble-Net Behavior (Exploitation Phase). There are two mechanisms utilized in order to mathematically model the bubble-net behavior. The first is the shrinking encircling mechanism that is given by decreasing the value of $a$ in equation (17) from 2 to 0 over the course of iterations. It should be pointed out that the fluctuation range of $A$ is also decreased by $a$. In other terms, the function range of $A$ is also decreased by $a$. In other words, $A$ is a random value that decreased from 2 to 0 . The new position of a search agent can be defined anywhere in between the original position of agent and the position of the current best agent by setting random values for $A$ in $[-1,1]$.

The second one is the spiral updating position to mimic the helix-shaped movement of humpback whales by using a spiral equation shown as follows:

$$
\begin{gathered}
B=\left|X^{*}(t)-X(t)\right|, \\
X(t+1)=X^{*}(t)+B \cdot e^{\mathrm{bk}} \cdot \cos (2 \pi \cdot k),
\end{gathered}
$$

where $b$ is a constant for determining the shape of the logarithmic spiral and $k$ is a random number in $[-1,1]$.

Whales swim around the prey in a sharking circle along a spiral-shaped path, and this behavior can be formulated mathematically as follows:

$$
X(t+1)= \begin{cases}X^{*}(t)-\mathrm{A} \cdot \mathrm{D}, & \text { if }(p<0.5), \\ X^{*}(t)+\mathrm{B} \cdot \mathrm{e}^{\mathrm{bk}} \cdot \cos (2 \pi \cdot k), & \text { if }(p \geq 0.5),\end{cases}
$$

where $p$ is a random number in $[0,1]$, which indicates that there is a probability of $50 \%$ to choose between the shrinking encircling mechanism and the spiral system. The source codes of the WOA algorithm we refer to are publicly available on http://www.alimirjalili.com/WOA.html. Algorithm 1 presents the pseudocode of the WOA algorithm [15]. It is notable that WOA can smoothly switch between the exploration and exploitation phases depending on only one parameter.

4.2.2. IWOA. It can be observed that the main advantage of WOA is that the mechanism of chasing the prey is realized by using the randomness of the best search agents and the employment of spiral to mimic the bubble-net attraction process of humpback whales. In addition, the WOA possesses few parameters to set and the most important one is self-turning along the iterative process. Because of these facts, WOA has two main drawbacks: (i) its adaptive parameter depends on the random distribution and (ii) it has a premature convergence. Many scholars improve the exploration and development capabilities of the algorithm. A chaotic whale optimization algorithm (CWOA) is proposed, which combines the features of standard WOA with chaotic maps to improve its performance to find the set of parameters that model solar cells [19]. An improved WOA based on social learning and wavelet mutation strategy designs a new linear incremental probability, which increases the possibility of global search [20]. 


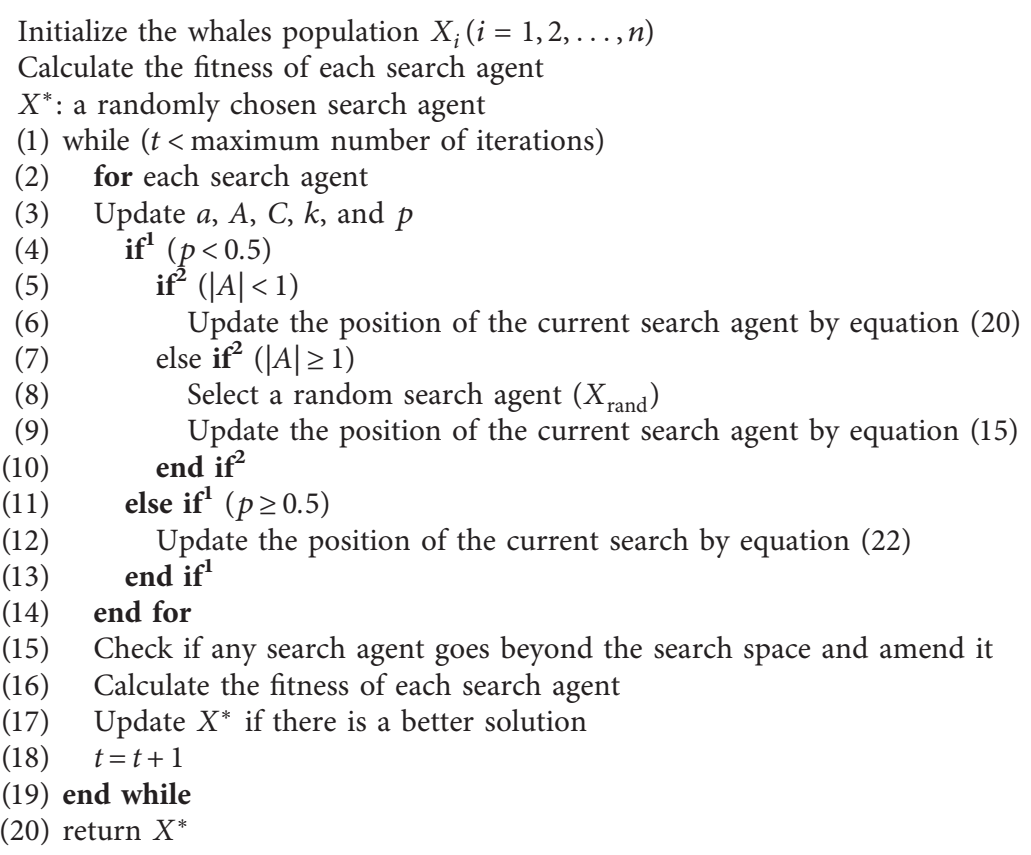

Algorithm 1: Pseudocode for WOA.

Actually, any swarm intelligent optimization algorithm has two different operations: global search (exploration is related to global search) and local search (exploitation is related to local search) in the iterative process $[21,22]$. As pointed out in Subsection 4.1, the transition between global search and local search in the optimizing process is generated by the value of $a$. A distance control parameter $a$ has a better global search ability to prevent the algorithm from falling into the local optimum, while a smaller $a$ has a stronger local search ability to increase the convergence speed of the algorithm.

It is notable that $a$ is the key to improving the performance of the algorithm. It decreases linearly from 2 to 0 in WOA. The actual search process of WOA is nonlinear and much more complicated, so the linear distance control parameter strategy cannot truly reflect it [16]. So researchers are increasingly paying close attention to improve the performance of WOA, especially distance control parameter $a[16,23]$. Actually, the algorithm is supposed to have a strong global search capability in the early stage of the search while maintaining a faster convergence rate, and it is ensured that the algorithm has a faster convergence rate in the later stage of the search [24]. Additionally, the excessive local search usually plunges into local optimum $[15,21]$. The proposed algorithm, which is called the improved whale optimization algorithm (IWOA), is based on the nonlinear convergence factor [25]. In the IOWA, a novel nonlinear update equation of convergence factor $(a)$ in (24) is designed to coordinate the abilities of exploration and exploitation.

$$
a=\left(a_{\text {initial }}-a_{\text {final }}\right)+\frac{\left(1-t / t_{\max }\right)}{\left(1-\mu \cdot t / t_{\max }\right)},
$$

where $a_{\text {initial }}$ is the initial value of the convergence factor of $a$. $a_{\text {final }}$ is the finial value of the convergence factor of $a . \mu$ is a nonlinear adjustment coefficient. $t$ is the current number of iterations. $t_{\max }$ is the maximum number of iterations. Then, the pseudocode of IWOA is presented in Algorithm 2.

According to Algorithms 1 and 2, the difference between WOA and IWOA is that a nonlinear update equation of convergence factor is redesigned in IWOA.

\section{Implementation and Results}

In this section, we present a case study of 2386 flight block times of China. We explain the original data at first. Later, evaluation metrics such as MSE, RMSE, MAE, MAPE, and Theil are used to compare the results obtained by the proposed approach with the standard SBTs published by CAAC in 2019. Finally, we analyze the fairness metric of airline generated by the standard reliability range and the optimal reliability range obtained by experiment.

5.1. Data Description. This research was supported by Operation Monitoring Center of CAAC. We collect the opinions (requested SBTs) of airlines through the civil aviation standard flight block time revision website platform on http://www.sinoflight.cn/, and airlines can only deal with their own. Each company organizes a coordinator to complete the flight block time application of flight routes operated by his company. We collected 22,768 opinions from 38 airlines, which include almost all civil airlines in China and 699,950 pieces of historical operating data from March 25, 2018, to March 30, 2019, including 2,386 flight block times. Figure 4 shows the proportion of airlines applying to revise standard SBTs. China Eastern airline is the airline with the largest number of requested standard SBTs, which accounts for $11.15 \%$, and China Southern airline 
Initialize the whales population $X_{i}(i=1,2, \ldots, n)$

Calculate the fitness of each search agent

$X^{*}$ : a randomly chosen search agent

(1) While ( $t \leq$ maximum number of iterations)

(2) for each search agent

(3) Update $a$ by equation (24)

(4) Update $A, C, k$, and $p$

(5) $\quad$ if $^{\mathbf{1}}(p<0.5)$

(6) $\quad$ if $^{2}(|A|<1)$

(7) Update the position of the current search agent by equation (20)

(8) else if ${ }^{2}(|A| \geq 1)$

(9) Select a random search agent $\left(X_{\text {rand }}\right)$

(10) Update the position of the current search agent by equation (15)

(11) $\quad$ end if ${ }^{2}$

(12) else if ${ }^{1}(p \geq 0.5)$

(13) Update the position of the current search by equation (22)

(14) end if ${ }^{1}$

(15) end for

(16) Check if any search agent goes beyond the search space and amend it

(17) Calculate the fitness of each search agent

(18) Update $X^{*}$ if there is a better solution

(19) $t=t+1$

(20) end while

(21) return $X^{*}$

Algorithm 2: Pseudocode for IWOA.

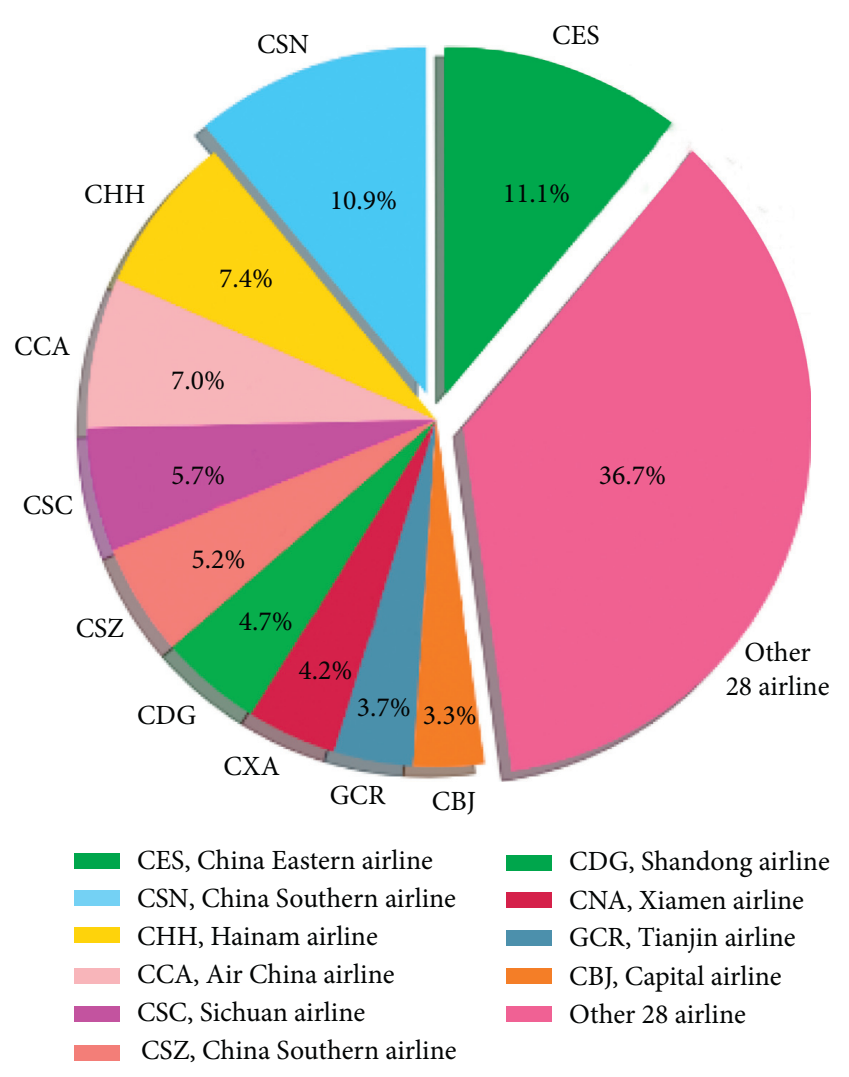

FIGURE 4: Proportion of airlines applying to revise standard SBTs.

accounts for $10.93 \%$, ranking second. All other 28 airlines accounted for $36.67 \%$ of the total standard SBTs. There are many flight block times that many airlines request to adjust and different airlines have different opinions. Figure 5 shows the fights routes with more than 6 airlines applying for adjustment at the same time. Figure 6 gives an example of flight routes with one airline requesting to adjust the time.

There are 22 flight routes where more than 6 airlines applied for the adjustment of flight block time as shown in Figure 5. CAAC needs to consider the flight times requested by the six airlines at the same time when adjusting these flight block time. Figure 6 shows the flight routes requested by Air China (CCA), $65.6 \%$ of which are flying to or from Beijing Capital International Airport (ZBAA). It is because CCA is one of the major airlines in China and its flight operations are based at ZBAA.

5.2. Standard Scheduled Block Time. The experiment results are obtained on an Intel Xeon E5-2620 with $2.10 \mathrm{GHz}$ and 16.0 GB RAM. The proposed IWOA, along with WOA, was programmed in Matlab (version 2018b) with reference to the original code at http://www.alimirjalili.com/WOA.html. Flight block time reliabilities are set in the range of $60-75 \%$ normally [4]. After initial experiments, they are tested using the same setting of parameters for fair comparisons among WOA and IWOA; that is, the maximum number of iterations is $100, n=30, b=1, a_{\text {initial }}=2, a_{\text {final }}=1$, and $\mu=25$ after initial experiments based on the research of $[15,25]$. Due to the stochastic nature of evolutionary algorithms, each algorithm with different reliability range is performed 20 times independently. The results are shown in Figure 7 and Table 2.

We use the most common evaluation metrics to measure optimal accuracy, and they are mean squared error (MSE), root mean square error (RMSE), mean absolute error 


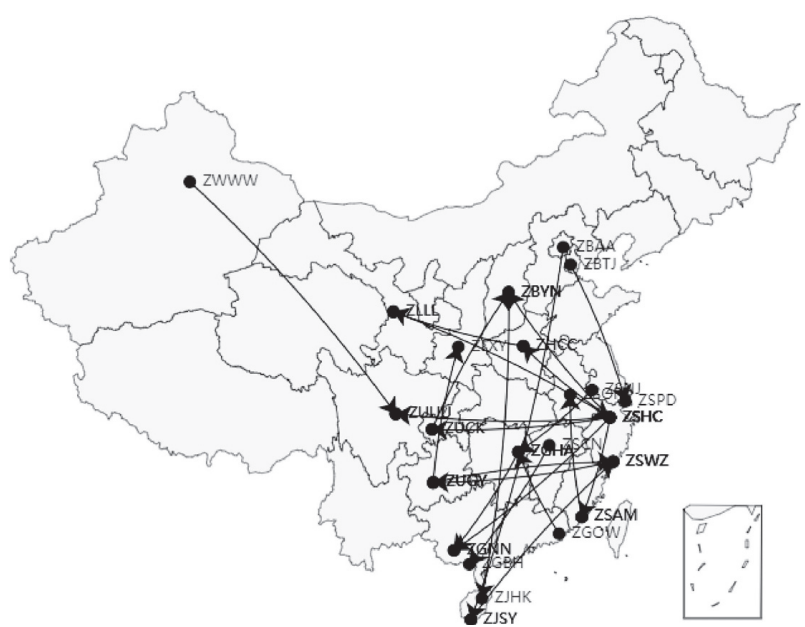

FIGURE 5: Flight routes of block time with more than 6 airlines applying for the adjustment.

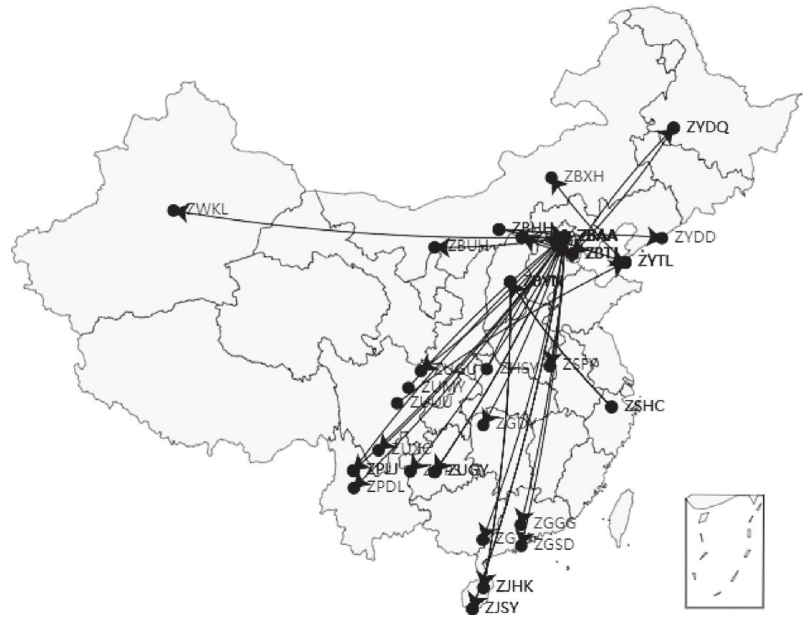

FIgUre 6: Flight routes requested by CCA for the adjustment of flight block time.

(MAE), mean absolute percentage error (MAPE), and Theil inequality coefficient (Theil) [26]. Let $\gamma^{g, j, r}$ denote the standard SBTs announced on the official website http://www. pre-flight.cn/ of 2019 on airport pair $g$, air season $j$, and aircraft type $r . K^{g, j, r}$ denotes the optimization of $\gamma^{g, j, k}$. Then define the forecast error as $e^{g, j, r}=\gamma^{g, j, r}-K^{g, j, r}$. We determine $\mathrm{MSE}=(1 / n) \sum\left(e^{g, j, r}\right)^{2}, \mathrm{RMSE}=\sqrt{(1 / n) \sum\left(e^{g, j, r}\right)^{2}}$, $\operatorname{MAE}=(1 / n) \sum\left|e^{g, j, r}\right|, \quad$ MAPE $=100 / n\left(\sum \sum\left|e^{g, j, r}\right| / \gamma^{g, j, r}\right)$, and $\quad$ Theil $=\sqrt{(1 / n) \sum\left(e^{g, j, r}\right)^{2}} / \sqrt{(1 / n) \sum\left(\gamma^{g, j, r}\right)^{2}}+$ $\sqrt{(1 / n) \sum\left(K^{g, j, r}\right)^{2}}$.

Figure 7 illustrates the convergence curves of WOA and IWOA. WOA took 560 seconds, while IWOA took 539 seconds in average. It can be clearly observed that the maximum distance between the fairness metric of an airline and the average fairness metrics of all airlines from two methods is remarkably different after a period of evolution. Figure 7 reveals that IWOA evolves fast and can obtain smaller fitness function value (lower maximum distance between the fairness metric of an airline and the average fairness metrics of all airlines). It shows that the average

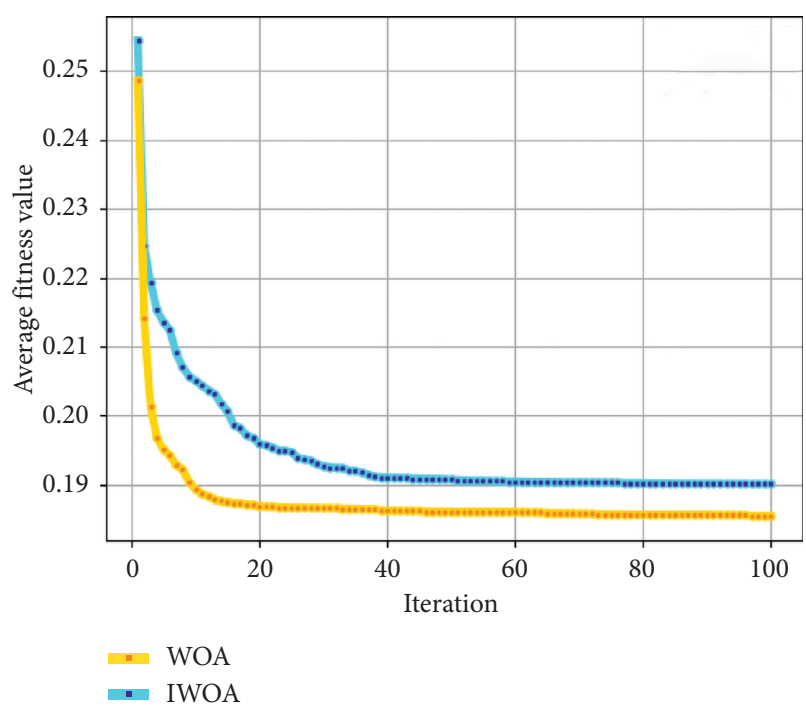

Figure 7: Convergence curves for WOA and IWOA.

TABLE 2: Evaluation metrics for WOA and IWOA with the reliability range $60 \%-75 \%$.

\begin{tabular}{lccccc}
\hline & MSE & RMSE & MAE & MAPE & Theil \\
\hline WOA & 359.5798 & 18.9281 & 13.9715 & 11.3934 & 0.0013 \\
IWOA & 333.0072 & 18.2043 & 13.7250 & 11.2294 & 0.0013 \\
\hline
\end{tabular}

fitness function values of WOA and IWOA are 0.1901 and 0.1866 , respectively. On average, WOA needs 40 generations to find the optimal result, while IWOA only needs 20 generations.

Table 2 shows the results of evaluation metrics for WOA and IWOA with scheduled flight block time of 2019 announced on the official website in the standard reliability range. The results of the proposed IWOA are very competitive. The values of MSA, RMSE, MAE, and MAPE for IWOA decrease by $7.97 \%, 3.97 \%, 1.80 \%$, and $1.46 \%$, respectively, compared to those for WOA. Therefore, IWOA performs better with the respect to the results of MSE, RMSE, MAE, and MAPE. The Theil value of both algorithms is 0.0013 . In conclusion, the optimal results of IWOA are better than those of WOA. Besides, the average fairness value for WOA is 0.9651 , while that for IWOA is 0.9817 .

Furthermore, we analyze the results of different flight block time reliabilities to investigate how the values of evaluation metrics change with the range of reliability. As discussed above, IWOA has been proven to be superior to WOA. Therefore, IWOA is chosen for further experiments; this paper selected the reliability in the ranges of $75 \%-90 \%$, $60 \%-70 \%, 70 \%-80 \%$, and $80 \%-90 \%$. Their running times are similar. The running time of reliability in the $75 \%-90 \%$ range is $485 \mathrm{~s}$, that in the $60 \%-70 \%$ range is $550 \mathrm{~s}$, that in the $70 \%-80 \%$ range is $534 \mathrm{~s}$, and that in the $80 \%-90 \%$ range is $556 \mathrm{~s}$. Figure 8 illustrates the evolutionary process of IWOA with different reliability range. Table 3 presents the results with different range of flight block time reliability, and Table 4 presents the results based on the air season and aircraft type. 


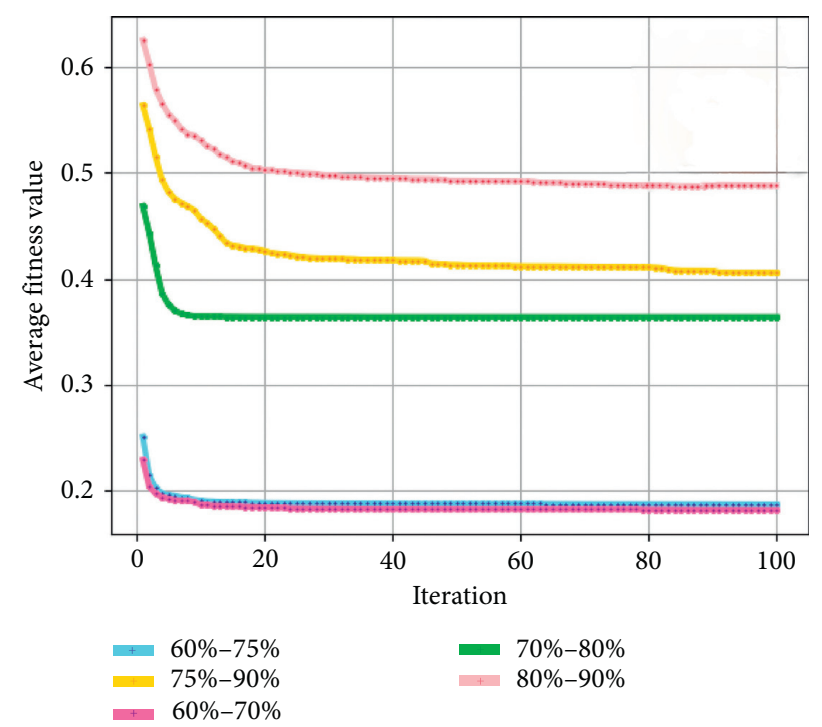

Figure 8: Convergence curves for different target block time reliability.

TABLE 3: Evaluation metrics of IWOA.

\begin{tabular}{lcccrr}
\hline Range of reliability & MSE & RMSE & MAE & MAPE & Theil \\
\hline $60 \%-75 \%$ & 333.0072 & 18.2043 & 13.7250 & 0.0013 \\
$75 \%-90 \%$ & 2425.7963 & 48.8482 & 21.8269 & 11.2294 & 17.8623 \\
$60 \%-70 \%$ & 308.8649 & 17.5407 & 13.3891 & 10.9601 & 0.0032 \\
$70 \%-80 \%$ & 906.9087 & 30.1147 & 18.8595 & 15.5608 & 0.0012 \\
$80 \%-90 \%$ & 2939.3689 & 53.8122 & 22.7504 & 18.6983 & 0.0020 \\
\hline
\end{tabular}

As illustrated in Figure 8, the reliability in the 60\%-75\% range has the smallest fitness function value. The obtained value of reliability in the $80 \%-90 \%$ range is the biggest among them. Obviously, smaller reliability leads to smaller maximum distance between the fairness metric of an airline and the average fairness metrics of all airlines in our problem. It can be seen that the results of reliability in the $70 \%-80 \%$ range can converge faster than others, while that of $75 \%-90 \%$ range converges the slowest. Results of reliability in the $70 \%-80 \%$ range converge at about 10 th iteration. However, the results of reliability in the $75 \%-90 \%$ range converge at about 80th iteration.

Evaluation metric values for different air seasons and aircraft types in 5 different ranges of reliability are compared in Tables 3 and 4 . Now, we present some specific observations regarding the results.

(i) It is obvious that the values of MSE, RMSE, MAE, MAPE, and Theil with the reliability in the 60\%$70 \%$ range are always the smallest. It is worth noting that the maximum distance of reliability in the $60 \%-75 \%$ range is not the smallest, which is bigger than that of reliability in the $60 \%-70 \%$ range in Figure 8.

(ii) By comparing the MSE, RMSE, MAE, MAPE, and Theil values of reliability in the $60 \%-75 \%$ range and those of reliability in the $75 \%-90 \%$ range, we can observe that the former are smaller than the latter. The values of MSE are sequentially reduced by
$24.00 \%, 3.61 \%, 73.75 \%$, and $9.80 \%$ under different pairings of aircraft types and air seasons.

(iii) It is interesting to find that the value of MSE with the reliability in the $70 \%-80 \%$ range of air season 7 , aircraft type 7 and air season 8 , aircraft type 8 is smaller than that of the reliability in the $80 \%-90 \%$ range, which is opposite to air season 7 , aircraft type 7 and air season 8, aircraft type 8. Maybe this is because the evaluation metrics are related to the amount of flight block times. There are very few companies operating flights with aircraft type 8 in China, accounting for only $6.92 \%$ of all flights, and the number of flight block times corresponding to this aircraft type is small.

5.3. Fairness Metric of Airlines. From the results of evaluation metrics in Section 5.2, it is observed that the values of MSE, RMSE, MAE, MAPE, and Theil of the reliability are always smaller in the $60 \%-70 \%$ range compared to 4 other ranges selected, thus making it the best reliability range among selected ones. We compared the airline fairness metric values in this reliability range against the standard reliability range. The mathematical expression of the proposed fairness metric is presented as equation (10) in Section 4.2. The results are shown in Figures 9 and 10 .

The average fairness metric values of the reliability in the $60 \%-75 \%$ and $60 \%-70 \%$ ranges are 0.9817 and 0.9920 , respectively, which are very close. There are 21 airlines whose 
TABLE 4: Evaluation metrics of IWOA based on the air season and aircraft type.

\begin{tabular}{|c|c|c|c|c|c|c|}
\hline & Range of reliability & MSE & RMSE & MAE & MAPE & Theil \\
\hline \multirow{5}{*}{$\begin{array}{l}\text { Air season } 7 \\
\text { Aircraft type } 7\end{array}$} & $60 \%-75 \%$ & 4402.8482 & 66.3525 & 50.9052 & 45.0407 & 0.0074 \\
\hline & $75 \%-90 \%$ & 5459.4110 & 73.8672 & 54.0402 & 49.2853 & 0.0078 \\
\hline & $60 \%-70 \%$ & 4402.7334 & 66.3516 & 50.8097 & 44.9590 & 0.0074 \\
\hline & $70 \%-80 \%$ & 5302.0306 & 72.8148 & 53.0805 & 48.1306 & 0.0078 \\
\hline & $80 \%-90 \%$ & 5608.4187 & 74.8729 & 54.6061 & 49.9786 & 0.0079 \\
\hline \multirow{5}{*}{$\begin{array}{l}\text { Air season } 7 \\
\text { Aircraft type } 8\end{array}$} & $60 \%-75 \%$ & 5338.9803 & 72.9717 & 59.6447 & 42.7891 & 0.0260 \\
\hline & $75 \%-90 \%$ & 5531.6979 & 74.2686 & 60.4354 & 45.7127 & 0.0252 \\
\hline & $60 \%-70 \%$ & 4944.7917 & 70.2449 & 57.1875 & 41.6076 & 0.0249 \\
\hline & $70 \%-80 \%$ & 5688.4271 & 75.4120 & 57.7479 & 46.2545 & 0.0242 \\
\hline & $80 \%-90 \%$ & 5454.2083 & 73.7803 & 59.6792 & 45.7779 & 0.0248 \\
\hline \multirow{5}{*}{$\begin{array}{l}\text { Air season } 8 \\
\text { Aircraft type } 7\end{array}$} & $60 \%-75 \%$ & 4880.3394 & 69.8584 & 54.1637 & 47.9007 & 0.0068 \\
\hline & $75 \%-90 \%$ & 8479.5075 & 91.8585 & 60.1777 & 54.1778 & 0.0083 \\
\hline & $60 \%-70 \%$ & 4899.4327 & 69.9949 & 54.2370 & 47.9743 & 0.0068 \\
\hline & $70 \%-80 \%$ & 5421.5231 & 73.6307 & 56.9631 & 51.0513 & 0.0069 \\
\hline & $80 \%-90 \%$ & 9433.5639 & 96.8662 & 61.1835 & 55.0664 & 0.0086 \\
\hline \multirow{5}{*}{$\begin{array}{l}\text { Air season } 8 \\
\text { Aircraft type } 8\end{array}$} & $60 \%-75 \%$ & 7369.6594 & 85.8059 & 66.4211 & 45.9281 & 0.0265 \\
\hline & $75 \%-90 \%$ & 8092.1471 & 89.7862 & 70.0216 & 49.8824 & 0.0264 \\
\hline & $60 \%-70 \%$ & 7272.3627 & 85.2502 & 65.5784 & 45.5530 & 0.0262 \\
\hline & $70 \%-80 \%$ & 9324.4510 & 96.5544 & 73.4863 & 53.1591 & 0.0273 \\
\hline & $80 \%-90 \%$ & 8373.4118 & 91.4033 & 70.7647 & 50.8655 & 0.0266 \\
\hline
\end{tabular}

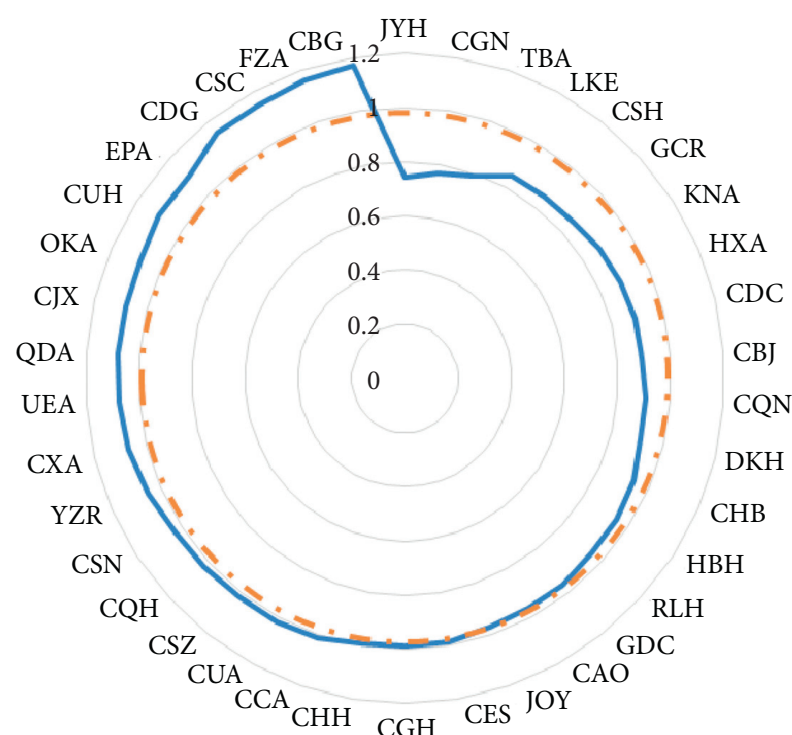

FIGURE 9: Fairness metric values of airlines with the reliability range of $60 \%-75 \%$.

fairness metric index values are not greater than 1 when the reliability range is $60 \%-75 \%$, which means that these airlines are treated fairly or preferentially as described in Section 4.1. The number of these preferential airlines is reduced to 19 in the range of $60 \%-70 \%$. As can be seen from Figure 9, the fairness metric values are concentrated between 0.8 and 1.2. Figure 10 presents that the fairness metric values are concentrated between 0.8 and 1.2, expect for one outlier point. This outlier point indicates that the metric value of JOY Air is 1.43. JOY Air is the most disfavored airline among 38 airlines. On the one hand, the deviation between the largest value and the smallest value of the reliability in the $60 \%-70 \%$

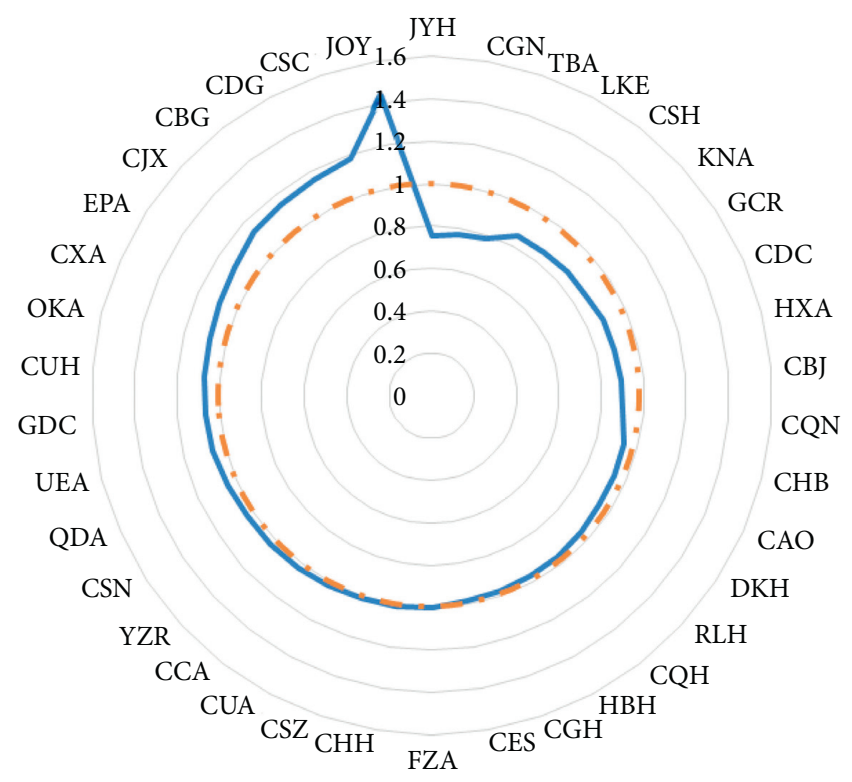

FIgURE 10: Fairness metric values of airlines with the reliability range of $60 \%-70 \%$.

range is larger than that in the $60 \%-75 \%$ range. On the other hand, the fairness metric value of more airlines is near 1 with the reliability in the $60 \%-70 \%$ range. Thus, we conclude that the value of the average fairness metric can be improved by sacrificing the most disfavored airlines when the reliability range is $60 \%-70 \%$.

\section{Conclusion}

This article focuses on the strategic standard SBTs for the next year under the regulation and guidelines of CAAC. We analyze the flight block time reliability with the standard 
SBTs increasing (or the actual flight block time decreasing) based on the historical flight data from March 25, 2018, to March 30, 2019. The objective of this paper is to develop and solve a novel strategic standard SBTs-setting model that simultaneously considers the fairness among airlines and the reliability of flight block time constraints. It generates and presents to all stakeholders information supporting the transparency of the decision-making process. Moreover, we improved WOA by using a novel nonlinear update equation of convergence factor. WOA and IWOA are employed to solve the model and generate the standard SBTs. By comparing the results of the two algorithms, we observed that IWOA evolves faster and could obtain lower maximum distance between the fairness metric of an airline and the average fairness metrics of all airlines. In addition, the values of evaluation metrics for the optimized model, such as MSE, RMSE, MAE, and MAPE of IWOA, are relatively smaller. Therefore, IWOA performs better than WOA.

To better understand how reliability constraints affect the SBTs, the proposed model is then applied to optimize standard SBTs under 5 different ranges of flight block time reliability in addition to the standard reliability range. From the results of evaluation metrics, it is seen that the values of MSE, RMSE, MAE, MAPE, and Theil of the reliability in the $60 \%-70 \%$ range are always the smallest. The values of evaluation metrics of the reliability in the $70 \%-80 \%$ and $80 \%-90 \%$ ranges are much bigger than those in the $60 \%-$ $70 \%$ range. The average fairness metric values of the reliability in the $60 \%-75 \%$ and $60 \%-70 \%$ ranges are 0.97 and 0.99 , respectively, which are very close. Besides, the average fairness of the reliability in the $60 \%-70 \%$ range is closer to 1 than that of the $60 \%-75 \%$ range. Based on the above findings, we suggest that the range of original standard reliability should be changed to $60 \%-70 \%$.

Our further research will be directed towards the weights of different flights considered during the standard SBTssetting process. Another research direction is to solve the standard SBTs-setting problem with other methodologies.

\section{Data Availability}

The data used to support this research article are available from the first author upon request.

\section{Conflicts of Interest}

The authors declare that there are no conflicts of interest regarding the publication of this paper.

\section{Acknowledgments}

This work was supported by the National Natural Science Foundation of China (Grant no. 61671237).

\section{References}

[1] Y. Wang, Y. Zhou, M. Hansen, and C. Chin, "Scheduled block time setting and on-time performance of U.S. and Chinese airlines-a comparative analysis," Transportation Research Part A: Policy and Practice, vol. 130, pp. 825-843, 2019.
[2] L. Kang and M. Hansen, "Behavioral analysis of airline scheduled block time adjustment," Transportation Research Part E: Logistics and Transportation Review, vol. 103, pp. 56-68, 2017.

[3] Y. Tian, Q. Wang, H. Li, and R. Vanga, "Assessment of flight block time reliability under different delay time windows: a case study," IEEE Access, vol. 8, pp. 9565-9577, 2020.

[4] L. Hao and M. Hansen, "Block time reliability and scheduled block time setting," Transportation Research Part B: Methodological, vol. 69, pp. 98-111, 2014.

[5] V. Deshpande and M. Arikan, "The impact of airline flight schedules on flight delays," Manufacturing \& Service Operations Management, vol. 14, no. 3, pp. 423-440, 2012.

[6] C. Mayer and S. Todd, Why do Airlines Systematically Schedule their Flights to Arrive Late, The Wharton School, University of Pennsylvania, Philadelphia, PA, USA, 2003.

[7] K. G. Zografos, M. A. Madas, and K. N. Androutsopoulos, "Increasing airport capacity utilisation through optimum slot scheduling: review of current developments and identification of future needs," Journal of Scheduling, vol. 20, no. 1, pp. 3-24, 2017.

[8] G. Andreatta and G. Lulli, "Equitable demand management strategies for different classes of customers," International Journal of Pure and Applied Mathematics, vol. 57, no. 1, pp. 1-22, 2009.

[9] P. Pellegrini, T. Bolić, L. Castelli, and R. Pesenti, "SOSTA: an effective model for the simultaneous optimisation of airport SloT allocation," Transportation Research Part E: Logistics and Transportation Review, vol. 99, pp. 34-53, 2017.

[10] K. G. Zografos and Y. Jiang, "A bi-objective efficiency-fairness model for scheduling slots at congested airports," Transportation Research Part C: Emerging Technologies, vol. 102, pp. 336-350, 2019.

[11] M. Redmond, M. C. Ann, and F. E. Jan, "The most reliable flight itinerary problem," Networks VPL, vol. 73, no. 3, pp. 325-343, 2019.

[12] M. A. P. Taylor, "Travel through time: the story of research on travel time reliability," Transportmetrica B: Transport Dynamics, vol. 1, no. 3, pp. 174-194, 2013.

[13] B. Manley and L. Sherry, "Analysis of performance and equity in ground delay programs," Transportation Research Part C: Emerging Technologies, vol. 18, no. 6, pp. 910-920, 2010.

[14] Q.-V. Pham, S. Mirjalili, N. Kumar, M. Alazab, and W.-J. Hwang, "Whale optimization algorithm with applications to resource allocation in wireless networks," IEEE Transactions on Vehicular Technology, vol. 69, no. 4, pp. 4285-4297, 2020.

[15] S. Mirjalili and A. Lewis, "The whale optimization algorithm," Advances in Engineering Software, vol. 95, pp. 51-67, 2016.

[16] M. Zhong and W. Long, "Whale optimization algorithm with nonlinear control parameter,"MATEC Web of Conferences, vol. 139, EDP Sciences, Les Ulis, France, 2017.

[17] A. S. Ahmed, M. A. Attia, N. M. Hamed, and A. Y. Abdelaziz, "Comparison between genetic algorithm and whale optimization algorithm in fault location estimation in power systems," in Proceedings of the 2017 Nineteenth International Middle East Power Systems Conference (MEPCON), IEEE, Cairo, Egypt, December 2017.

[18] P. D. P. Reddy, V. C. V. Reddy, and T. G. Manohar, "Whale optimization algorithm for optimal sizing of renewable resources for loss reduction in distribution systems," Renewables: Wind, Water, and Solar, vol. 4, no. 1, pp. 2-13, 2017.

[19] D. Oliva, M. Abd El Aziz, and A. Ella Hassanien, "Parameter estimation of photovoltaic cells using an improved chaotic 
whale optimization algorithm," Applied Energy, vol. 200, pp. 141-154, 2017.

[20] W. Y. Guo, T. Liu, F. Dai et al., "An improved whale optimization algorithm for forecasting water resources demand," Applied Soft Computing, vol. 86, 2020.

[21] Y. Yang, B. Yang, S. Wang, W. Liu, and T. Jin, "An improved grey wolf optimizer algorithm for energy-aware service composition in cloud manufacturing," The International Journal of Advanced Manufacturing Technology, vol. 105, no. 7-8, pp. 3079-3091, 2019.

[22] J. Chen, B. Xin, Z. Dou, L. Dou, and J. Zhang, "Optimal contraction theorem for exploration-exploitation tradeoff in search and optimization," IEEE Transactions on Systems, Man, and Cybernetics-Part A: Systems and Humans, vol. 39, no. 3, pp. 680-691, 2009.

[23] H. F. Eid and A. Abraham, "Adaptive feature selection and classification using modified whale optimization algorithm," International Journal of Computer Information Systems and Industrial Management Applications, vol. 10, pp. 174-182, 2018.

[24] Y. Shi and R. C. Eberhart, "Empirical study of particle swarm optimization," in Proceedings of the 1999 Congress on Evolutionary Computation-CEC99, vol. 3, IEEE, Washington, DC, USA, July 1999.

[25] W. Long, S. Cai, J. J. Jiao et al., "Improved whale optimization algorithm for large scale optimization problems," Systems Engineering Theory \& Practice, vol. 37, no. 11, pp. 2983-2994, 2017.

[26] R. J. Hyndman and A. B. Koehler, "Another look at measures of forecast accuracy," International Journal of Forecasting, vol. 22, no. 4, pp. 679-688, 2006. 